\title{
Copeptin as a Prognostic Marker in Acute Chest Pain and Suspected Acute Coronary Syndrome
}

\author{
Beata Morawiec $(D),{ }^{1}$ Damian Kawecki, ${ }^{1}$ Brygida Przywara-Chowaniec, ${ }^{1}$ Mariusz Opara, ${ }^{1}$ \\ Piotr Muzyk, ${ }^{1}$ Lam Ho, ${ }^{2}$ Lui Chun Tat, ${ }^{3}$ Artur Gabrysiak, ${ }^{1}$ Olivier Muller, ${ }^{4}$ \\ and Ewa Nowalany-Kozielska ${ }^{1}$
}

\author{
${ }^{1}$ 2nd Department of Cardiology, School of Medicine with the Division of Dentistry in Zabrze, Medical University of Silesia, Katowice, \\ M. Skłodowskiej-Curie 10, 41-800 Zabrze, Poland \\ ${ }^{2}$ Department of Cardiology, Tuen Mun Hospital, 23 Tsing Chung Koon Rd, Tuen Mun, Hong Kong \\ ${ }^{3}$ Department of Accident and Emergency, Tuen Mun Hospital, 23 Tsing Chung Koon Rd, Tuen Mun, Hong Kong \\ ${ }^{4}$ Department of Cardiology, Lausanne University Hospital, rue du Bugnon 46, 1011 Lausanne, Switzerland
}

Correspondence should be addressed to Beata Morawiec; beamorawiec@wp.pl

Received 7 June 2017; Revised 25 August 2017; Accepted 18 October 2017; Published 24 January 2018

Academic Editor: Salvatore Di Somma

Copyright ( 2018 Beata Morawiec et al. This is an open access article distributed under the Creative Commons Attribution License, which permits unrestricted use, distribution, and reproduction in any medium, provided the original work is properly cited.

Background. In patients admitted with chest pain and suspected acute coronary syndrome (ACS), it is crucial to early identify those who are at higher risk of adverse events. The study aim was to assess the predictive value of copeptin in patients admitted to the emergency department with chest pain and nonconclusive ECG. Methods. Consecutive patients suspected for an ACS were enrolled prospectively. Copeptin and high-sensitive troponin T (hs-TnT) were measured at admission. Patients were followed up at six and 12 months for the occurrence of death and major adverse cardiac and cerebrovascular events (MACCE). Results. Among 154 patients, 11 patients died and 26 experienced MACCE. Mortality was higher in copeptin-positive than copeptinnegative patients with no difference in the rate of MACCE. Copeptin reached the AUC 0.86 (0.75-0.97) for prognosis of mortality at six and $0.77(0.65-0.88)$ at 12 months. It was higher than for hs-TnT and their combination at both time points. Copeptin was a strong predictor of mortality in the Cox analysis (HR14.1 at six and HR4.3 at 12 months). Conclusions. Copeptin appears to be an independent predictor of long-term mortality in a selected population of patients suspected for an ACS. The study registration number is ISRCTN14112941.

\section{Introduction}

The optimal risk stratification in patients with acute coronary syndrome (ACS) without persistent ST segment elevation is a priority for clinicians. Patients after an ACS remain on higher risk of the development of heart failure, further ischemic events, and death. It is crucial to identify possibly early those subjects who present with chest pain and are at higher risk, in order to enable the introduction of adequate strategy in optimal time frames. The guidelines for diagnosis and treatment of non-ST segment elevation ACS focus attention on early stratification of short- and long-term risk [1]. However, cardiac troponin (cTn), despite being the gold standard marker for diagnosis of myocardial injury, remains suboptimal in terms of early risk assessment. Due to the character of the release profile, repetitive blood sampling for $\mathrm{cTn}$ is needed to achieve satisfactory prognostic accuracy [2]. The introduction of high-sensitive assays allowed earlier stratification of patients but did not justify single measurement or risk stratification based solely on these assays. While looking for complemental markers that would increase the accuracy of cTn, we face a large variety of biomarkers [3]. It is of utmost importance from a practical point of view to indicate those that could be used for diagnostic and prognostic purposes simultaneously as early as possible after symptom onset. Copeptin, the C-terminal part of the prohormone for vasopressin, is a marker of acute endogenous stress, with rapid release pattern [4]. Several previous studies showed 
its good diagnostic accuracy in patients with an ACS [5-7]. Its potential prognostic role was described in patients with the history of an acute myocardial infarction (AMI) who developed heart failure $[6,8]$. The strategy of combined use of copeptin and cTn, besides serving as a diagnostic tool, could identify patients with chest pain at higher risk for adverse events and the development of heart failure (HF) very early after symptom onset, thus enabling early triage decision. Despite the combination of copeptin and highsensitive Tn (hs-Tn) was extensively described in terms of diagnosis of an ACS, there is insufficient data regarding its prognostic accuracy, especially in patients without persistent ST segment elevation. We therefore aimed to investigate if copeptin provides information on the prediction of outcome in patients admitted to tertiary cardiac centers with symptoms of ACS and nonconclusive ECG.

\section{Methods}

The Copeptin for Acute Coronary Syndrome (COPACS) is a prospective, investigator-initiated, observational study with the aim to evaluate the role of copeptin in the diagnostic and prognostic process in patients with acute chest pain to enhance rapid evaluation in the emergency department.

Details on the design and the chart of the study were widely described previously [9]. In brief, consecutive patients presenting to the emergency department of the 2nd Department of Cardiology in Zabrze, Medical University of Silesia, Katowice, Poland, were screened in 24/7 manner. Patients with chest pain lasting 5 minutes or more during the last 6 hours and the absence of persistent ST segment elevation in admission ECG were prospectively enrolled to the study. Major conditions with proved influence on copeptin elevation were regarded as exclusion criteria (e.g., end-stage renal disease, sepsis, anaemia, and hyponatremia). According to the tertiary character of the enrolling center, patients admitted to the emergency department conform a highly preselected population, referred mainly via regional hospital. In the structure of our health care system, high rate of patients with chest pain and without persistent ST segment elevation on ECG undergoes initial stratification in regional hospitals. Therefore, patients are referred and admitted to tertiary cardiac centers after initial qualification to invasive coronary angiography with longer delays from chest pain onset and high suspicion of an ACS. As an effect, first, vast majority of patients present later than six hours after chest pain onset and, second, high rate of patients is finally diagnosed with acute coronary syndrome.

The study protocol conforms to the ethical guidelines of the 1975 Declaration of Helsinki as reflected in a priori approval by the Ethical Committee of Medical University of Silesia (decision number KNW/0022/KB1/187/11). All patients gave their written informed consent before inclusion to the study. The study registration number is ISRCTN14112941.

The design of the study, data gathering, and analysis were conducted according to the STARD guidelines for studies of diagnostic/prognostic accuracy. All authors contributed to the work by participating in the design, collecting and analyzing the data, and writing the paper, and all accepted the final draft of the manuscript.

After inclusion, each patient underwent initial clinical examination which included physical examination, 12-lead electrocardiogram (ECG), echocardiographic examination, and standard laboratory tests (blood count, sodium, potassium, creatinine, GFR, C-reactive protein [CRP], and Nterminal pro-B type natriuretic peptide [NT-proBNP]). $\mathrm{Hs}-\mathrm{TnT}$, creatine kinase myocardial bound (CK-MB), and copeptine were measured at admission (T0). Copeptin was double-blinded until final adjudication of the diagnosis. $\mathrm{Hs}-\mathrm{TnT}$ and CK-MB were afterwards measured at six hours (T6) and repeated according to clinical indications.

Initial diagnosis was set by the emergency physician and was verified by a supervisor cardiologist based on available data and the ESC guidelines [1]. All patients were stratified according to the GRACE 1.0 risk score.

Further, all included patients underwent routine diagnostic and therapeutic procedures as indicated in the ESC guidelines for non-ST segment elevation ACS [1] and according to the study design [9]. Final diagnosis of non-ST elevation myocardial infarction (NSTEMI), unstable angina (UA), or other causes of chest pain was set based on independent opinions of two cardiologists, after analysis of all available data and tests gathered during the hospital stay. In case of incoherence of their diagnosis, a third cardiologist was asked for opinion.

Copeptin was measured once, at admission (T0) from the blood sample managed according to the instructions of the manufacturer of the test. The measurement was performed using the BRAHMS Copeptin KRYPTOR kit on BRAHMS KRYPTOR compact plus analyzer (BRAHMS GmbH, Hennigsdorf, Germany)-detection limit at 4.8-500 pmol/1, 20\% coefficient of variation $(\mathrm{CV})$ at $12 \mathrm{pmol} / \mathrm{l}$, and the 97.5 th percentile for healthy population at $17.4 \mathrm{pmol} / \mathrm{l}$. According to the general rule for the optimal cutoff for a marker at the 99th percentile of healthy population, copeptin was regarded as positive when $\geq 17.4 \mathrm{pmol} / \mathrm{l}$, following available information provided by the manufacturer on the most compliant value (97.5th percentile) to that recommended in the guidelines $[1,10]$. For secondary analysis, the concentration of $10 \mathrm{pmol} / \mathrm{l}$ was used as a cutoff [10].

Troponin $\mathrm{T}$ was measured at admission (T0), after 6 hours from admission (T6), and at further time points according to the decision of treating physician. Troponin $\mathrm{T}$ was measured using an Elecsys Troponin $\mathrm{T}$ hs STAT kit on a cobas e 411 analyzer (Roche Diagnostics $\mathrm{GmbH}$, Mannheim, Germany) with a high-sensitive electrochemiluminescence method (limit of detection 3-10,000 ng/l, 99th percentile for healthy population $14 \mathrm{ng} / \mathrm{l}$ [95\% CI 12.7 $24.9 \mathrm{ng} / \mathrm{l}]$, and $10 \% \mathrm{CV} 3 \mathrm{ng} / \mathrm{l})$. Hs-TnT was regarded as positive when $\geq 14 \mathrm{ng} / \mathrm{l}$, according to the manufacturer's indications and the guidelines [1].

The observational data of all patients were analyzed after six months and one year. Six-month follow-up was conducted in a phone call with the patient, relatives, or primary care physician and included the information on Canadian Cardiovascular Society (CCS) class, New York Heart Association (NYHA) class, and the occurrence of endpoints. At one 
year, during a visit in the outpatient unit, following data were gathered: CCS and NYHA classes, the occurrence of endpoints, echocardiogram with the assessment of EF, and blood draw for NT-proBNP.

Primary endpoint was defined as death of cardiovascular origin. Secondary endpoints were major adverse cardiac and cerebrovascular events (MACCE) combined with death of cardiovascular origin, nonfatal AMI, UA, repeated cardiac revascularization, and stroke. Patients were also screened for the occurrence of major bleeding.

Maximal hs-TnT/CK-MB was the maximal concentration of the biomarker measured during the hospital stay. Smoker was regarded as past if one was free of smoking for at least one year before admission. Familial history of coronary artery disease was positive if AMI, stroke, or cardiac death occurred in at least one first-degree female relative at the age of $<55$ years or male relative at the age of $<65$ years. Coronary artery disease was diagnosed in coronary angiogram if the stenosis of coronary artery was $>75 \%$ (or $>50 \%$ for left main).

Data were checked for normality of distribution with the Shapiro-Wilk test. Continuous variables are presented as mean, standard deviation (SD), or median (interquartile range [IQR]) and were compared with the Student $t$-test or Mann-Whitney test and ANOVA or Kruskal-Wallis test, depending on their distribution. Categorical variables are presented as $n(\%)$ and were compared with chi-square test. The correlation between copeptin and other parameters was assessed with Spearman's method. To evaluate the prognostic accuracy, the receiver operating characteristic (ROC) curves with areas under the curve (AUC) were used and compared with the $z$ test. Survival curves for copeptin and hs$\mathrm{Tn} \mathrm{T}$ were performed with the Kaplan-Meier method. The influence of biomarkers and preselected baseline, clinical, and procedural parameters on the occurrence of endpoints was calculated in the Cox proportional hazard regression model. The taxonomic analysis (focus analysis) was used as a supplementary method to analyze prognostic accuracy in a multivariate manner and is described in online Supplementary Material 1 . The $p$ value of $<0.05$ was assumed significant throughout all analyses. All analyses were performed with Statistica software, version 10PL (StatSoft Inc., Tulsa, OK, USA); GraphPad Prism, version 6.00 (GraphPad, La Jolla, California, USA); and platform R, version 3.0.2 (R Foundation for Statistical Computing, Vienna, Austria).

\section{Results}

During the study period, a total of 1665 were screened. After exclusion of 424 patients presented with STEMI and 1241 patients due to other exclusion criteria (40 patients with anaemia, 31 with hyponatremia, 13 with laboratory errors, 995 patients with chest pain out of inclusion criteria, and 8 patients due to withdrawal of the informed consent), a total of 154 patients were enrolled to the study. Mean age of the population was 63 , SD was 12 years, and $65 \%$ were male. After adjudication of the final diagnosis, 105 patients (68\%) were diagnosed with NSTEMI, 30 patients $(20 \%)$ were diagnosed with UA, and 19 patients (12\%) presented other causes of chest pain. Mean GRACE risk score was 124 (104-147) for the population. Copeptin was positive in 48 patients (31\%) with the median of 11.56 (5.67-21.61) pmol/l.

Patients positive and negative for copeptin had a similar clinical profile. Groups differed significantly with age; patients were older in the copeptin-positive group. Regarding the risk profile, patients positive for copeptin had higher hs-TnT concentrations measured at every time point; higher six-hour and maximal concentration of CK-MB; higher admission NT-proBNP, CRP, and leukocytosis and lower GFR; and higher GRACE risk score. Patients with positive copeptin result had similar procedural characteristics and received equal medical therapy as copeptin-negative patients with significant differences with regard to in-hospital catecholamine administration, more frequent in copeptinpositive patients. Results are summarized in Table 1. Similar results were observed for patients positive and negative for copeptin with the cutoff $10 \mathrm{pmol} / \mathrm{l}$ and are presented in Supplemental Table S1.

Positive correlation of copeptin was found with age $(r=0.36$ [0.18-0.52]), maximal hs-TnT $(r=0.24$ [0.05$0.42])$, six-hour and maximal CK-MB $(r=0.34[0.16-0.5]$ and $r=0.35$ [0.17-0.51], resp.), leukocytosis $(r=0.36$ [0.18-0.52]), and the GRACE risk score $(r=0.48$ [0.32$0.62])$. Negative correlation reached statistical significance with GFR $(r=-0.27[-0.44 ;-0.08])$ and $\mathrm{EF}(r=-0.3$ $[-0.46 ;-0.11])$.

Follow-up was completed in $98 \%$ of patients at six months and $95 \%$ of patients at one year. Overall, 11 patients died (8\%) and $26(18 \%)$ experienced MACCE (11 deaths, 3 AMI, 10 PCI, and 2 strokes). No major bleeding was reported during the follow-up. Patients who died had significantly higher concentrations of copeptin than survivors (103 [21$168] \mathrm{pmol} / \mathrm{l}$ versus 11 [5.3-20] pmol/l, resp., $p=0.001$ ), while there was no statistically significant difference in copeptin level between patients who experienced MACCE and other patients (17 [6.0-52] pmol/l versus 11 [5.4-20] pmol/l, resp., $p=0.19)$.

At one year, copeptin-positive patients had higher NTproBNP concentrations than copeptin-negative patients (216 [140-457] $\mathrm{pg} / \mathrm{ml}$ versus 147 [80-359] $\mathrm{pg} / \mathrm{ml}, p=0.049$ ). They also had higher NYHA class with more patients in class III (20\% versus $8 \%)$ and less in class I (50\% versus $68 \%)$ than copeptin-negative patients $(p=0.014)$. Concentrations of NT-proBNP remained higher in copeptin-positive than in copeptin-negative patients when the cutoff $10 \mathrm{pmol} / \mathrm{l}$ was used (250 [118-567] $\mathrm{pg} / \mathrm{ml}$ versus 135 [77-227] pg/ml, $p=0.003)$, and no statistically significant difference was observed in NYHA class between both groups $(p=0.10)$.

According to Kaplan-Meier analysis, patients with negative copeptin levels had significantly better survival than copeptin-positive patients at six months (103/104 versus $41 / 47$ patients, $p=0.001)$ and one year (98/102 versus 38/ 45 patients, $p=0.011$ ) (Figure $1(\mathrm{a})$ ). After dichotomization with the cutoff $10 \mathrm{pmol} / \mathrm{l}$, similar results were observed at six months $(69 / 71$ versus 67/76, $p=0.033)$ and one year (71/71 versus $73 / 80$ patients, $p=0.011$ ) (Supplemental Figure S1). No statistically significant difference in survival was observed regarding hs-TnT levels throughout the 
TABLE 1: Baseline characteristics in patients positive and negative for copeptin.

\begin{tabular}{|c|c|c|c|c|}
\hline & $\begin{array}{l}\text { Overall cohort } \\
\quad(n=154)\end{array}$ & $\begin{array}{c}\text { Copeptin }<17.4 \mathrm{pmol} / \mathrm{l} \\
(n=106)\end{array}$ & $\begin{array}{c}\text { Copeptin } \geq 17.4 \mathrm{pmol} / 1 \\
(n=48)\end{array}$ & $p$ value \\
\hline \multicolumn{5}{|c|}{ Baseline parameters and medical history } \\
\hline Age (years) & $63(57-73)$ & $62(56-69)$ & $65(57-78)$ & 0.04 \\
\hline Male sex & $100,65 \%$ & $72,65.5 \%$ & $28,58.3 \%$ & 0.25 \\
\hline BMI $\left(\mathrm{kg} / \mathrm{m}^{2}\right)$ & $28.7(42.9-32.3)$ & $28(25-32)$ & $29(25-32)$ & 0.93 \\
\hline $\mathrm{CAD}$ & $67,44 \%$ & $50,45.5 \%$ & $17,35.4 \%$ & 0.17 \\
\hline Hypertension & $114,74 \%$ & $75,68.2 \%$ & $39,81.3 \%$ & 0.17 \\
\hline Diabetes mellitus & $42,27 \%$ & $28,25.5 \%$ & $14,29.2 \%$ & 0.72 \\
\hline PAD & $4,2.6 \%$ & $3,2.7 \%$ & $1,2.1 \%$ & 0.79 \\
\hline Familial history of CAD & $21,14 \%$ & $18,16.4 \%$ & $3,6.3 \%$ & 0.07 \\
\hline Current smoker & $51,33 \%$ & $38,34.5 \%$ & $13,27.1 \%$ & 0.28 \\
\hline Past smoker & $31,20 \%$ & $23,20.9 \%$ & $8,16.7 \%$ & 0.47 \\
\hline Dyslipidemia & $67,44 \%$ & $49,44.5 \%$ & $18,37.5 \%$ & 0.31 \\
\hline History of AMI & $46,30 \%$ & $36,32.7 \%$ & $10,20.8 \%$ & 0.10 \\
\hline History of PCI & $48,31 \%$ & $36,32.7 \%$ & $12,25.0 \%$ & 0.27 \\
\hline History of CABG & $7,4.5 \%$ & $7,6.4 \%$ & $0,0 \%$ & 0.07 \\
\hline History of stroke & $4,2.6 \%$ & $3,2.7 \%$ & $1,2.1 \%$ & 0.79 \\
\hline \multicolumn{5}{|c|}{ Baseline clinical status } \\
\hline Heart rate (beats/min) & $75(66-88)$ & $70(65-80)$ & $75(70-85)$ & 0.07 \\
\hline Systolic BP & $140(123-160)$ & $140(125-160)$ & $140(120-160)$ & 0.54 \\
\hline $\mathrm{EF}(\%)$ & $55(45-60)$ & $55(45-60)$ & $55(43-60)$ & 0.56 \\
\hline NYHA class III or IV & $4,2.6 \%$ & $1,0.9 \%$ & $3,6.3 \%$ & 0.06 \\
\hline Killip class & & & & 0.50 \\
\hline 1 & $139,90 \%$ & $98,89.1 \%$ & $41,85.4 \%$ & \\
\hline 2 & $14,9.1 \%$ & $7,6.4 \%$ & $7,14.6 \%$ & \\
\hline 3 & $1,0.6 \%$ & $1,0.9 \%$ & $0,0 \%$ & \\
\hline 4 & $0,0 \%$ & $0,0 \%$ & $0,0 \%$ & \\
\hline GRACE & $124(104-146)$ & $120(101-141)$ & $131(111-167)$ & 0.03 \\
\hline \multicolumn{5}{|c|}{ Laboratory parameters } \\
\hline Hs-TnT T0 (ng/l) & $33(13-143)$ & $25(11-125)$ & $68(30-177)$ & 0.01 \\
\hline Hs-TnT T6 (ng/l) & $75(16-397)$ & $32(13-247)$ & $234(43-2284)$ & $<0.001$ \\
\hline Hs-TnT max (ng/l) & $105(23-530)$ & $52(17-274)$ & $236(74-2070)$ & $<0.001$ \\
\hline CK-MB T0 (IU/l) & $20(15-30)$ & $19(14-28)$ & $22(17-36)$ & 0.06 \\
\hline CK-MB T6 (IU/l) & $21(14-45)$ & $18(13-32)$ & $33(18-107)$ & $<0.001$ \\
\hline CK-MB max (IU/l) & $27(18-53)$ & $25(17-42)$ & $42(25-111)$ & $<0.001$ \\
\hline NT-proBNP (pg/ml) & $350(163-1074)$ & $289(150-872)$ & $491(223-1979)$ & 0.02 \\
\hline $\mathrm{CRP}(\mathrm{mg} / \mathrm{l})$ & $2.9(1.3-5.5)$ & $3(1-5)$ & $4(2-7)$ & 0.03 \\
\hline Leukocytosis $\left(10^{3} / \mu \mathrm{l}\right)$ & $8.4(6.9-10.3)$ & $8(6-9)$ & $10(8-12)$ & $<0.001$ \\
\hline Hemoglobin (g/dl) & $14(13-15)$ & $14(13-15)$ & $14(13-15)$ & 0.34 \\
\hline $\operatorname{GFR}\left(\mathrm{ml} / \mathrm{min} / 1.73 \mathrm{~m}^{2}\right)$ & $92(76-110)$ & $93(77-115)$ & $90(67-99)$ & 0.08 \\
\hline \multicolumn{5}{|c|}{ In-hospital parameters } \\
\hline Diagnosis of CAD & $116,75 \%$ & $83,75.5 \%$ & $33,68.8 \%$ & 0.11 \\
\hline Medical therapy & $38,25 \%$ & $25,22.7 \%$ & $13,27.1 \%$ & 0.64 \\
\hline PCI & $90,58 \%$ & $60,54.5 \%$ & $30,62.5 \%$ & 0.49 \\
\hline CABG & $33,21 \%$ & $25,22.7 \%$ & $8,16.7 \%$ & 0.33 \\
\hline Catecholamines & $4,2.6 \%$ & $0,0 \%$ & $4,8.3 \%$ & 0.003 \\
\hline ASA & $141,92 \%$ & $97,88.2 \%$ & $44,91.7 \%$ & 0.79 \\
\hline DAPT & $99,64 \%$ & $68,61.8 \%$ & $31,64.6 \%$ & 0.96 \\
\hline$\beta$-Blocker & $134,87 \%$ & $93,84.5 \%$ & $41,85.4 \%$ & 0.81 \\
\hline
\end{tabular}


TABLE 1: Continued.

\begin{tabular}{|c|c|c|c|c|}
\hline & $\begin{array}{l}\text { Overall cohort } \\
\quad(n=154)\end{array}$ & $\begin{array}{c}\text { Copeptin }<17.4 \mathrm{pmol} / \mathrm{l} \\
(n=106)\end{array}$ & $\begin{array}{c}\text { Copeptin } \geq 17.4 \mathrm{pmol} / \mathrm{l} \\
(n=48)\end{array}$ & $p$ value \\
\hline ACE inhibitor & $126,82 \%$ & $91,82.7 \%$ & $35,72.9 \%$ & 0.07 \\
\hline Statin & $135,88 \%$ & $95,86.4 \%$ & $40,83.3 \%$ & 0.26 \\
\hline Diuretic & $45,29 \%$ & $31,28.2 \%$ & $14,29.2 \%$ & 0.97 \\
\hline Ca-blocker & $39,25 \%$ & $27,24.5 \%$ & $12,25.0 \%$ & 0.98 \\
\hline Nitroglycerin & $17,11 \%$ & $12,10.9 \%$ & $5,10.4 \%$ & 0.89 \\
\hline \multicolumn{5}{|c|}{ Final diagnosis } \\
\hline Unstable angina & $30,20 \%$ & $27,24.5 \%$ & $3,6.3 \%$ & 0.005 \\
\hline NSTEMI & $105,68 \%$ & $65,59.1 \%$ & $40,83.3 \%$ & 0.007 \\
\hline
\end{tabular}

Data presented as $n, \%$, or median (25th-75th interquartile range). ACE: angiotensin-converting enzyme; AMI: acute myocardial infarction; ASA: acetylsalicylic acid; BMI: body mass index; BP: blood pressure; CABG: coronary artery bypass grafting; CAD: coronary artery disease; CK-MB: creatine kinase myocardial bound; CRP: C-reactive protein; DAPT: dual antiplatelet treatment; EF: ejection fraction; GFR: glomerular filtration ratio; GRACE: Global Registry for Acute Coronary Events; Hs-TnT: high-sensitive troponin T; NSTEMI: non-ST segment elevation myocardial infarction; NT-proBNP: N-terminal pro-B-type natriuretic peptide; NYHA: New York Heart Association; PAD: peripheral artery disease; PCI: percutaneous coronary intervention.

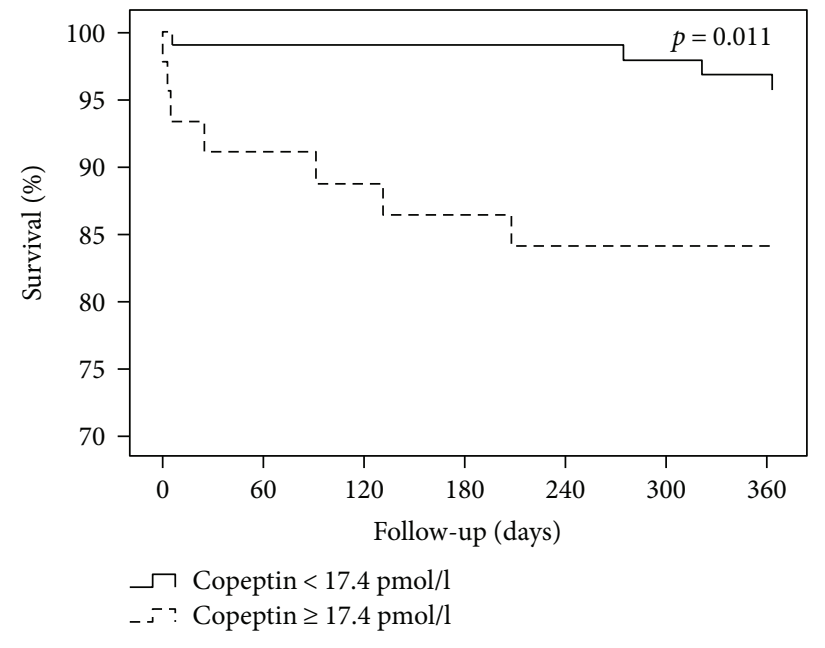

(a)

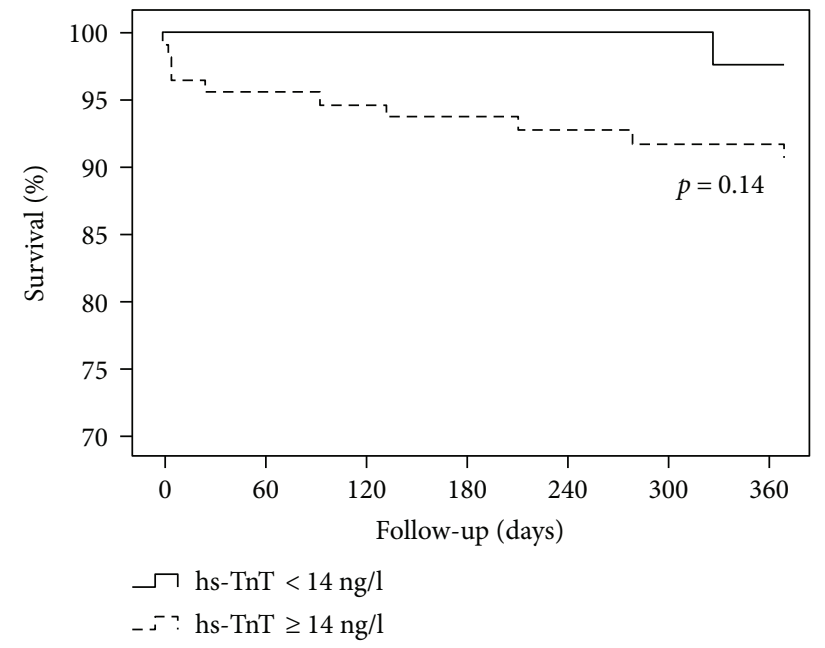

(b)

FIgURE 1: Survival curves or copeptin (a) and hs-TnT (b). Hs-TnT: high-sensitive troponin T.

follow-up ( $p=0.10$ and $p=0.14$ at six months and one year, resp.) (Figure 1(b)).

Copeptin achieved a good prognostic accuracy in the ROC analysis at six months (AUC 0.86 [0.75-0.97], $p<0.001$ ) and one year (AUC 0.77 [0.65-0.88], $p=0.003$ ). It was higher when compared to hs-TnT and their combination at six months (0.62 [0.53-0.72], $p=0.002$, versus 0.76 [0.53-0.99], $p=0.42$, resp.) and one year (0.63 [0.52-0.75], $p=0.12$, versus $0.63[0.43-0.83], p=0.24$, resp.). Of note, the ROC analysis of hs-TnT did not reach statistical significance for the prediction of death at any time point. Additional analysis with the GRACE risk score for six-month prediction of death revealed that copeptin was not inferior to the GRACE risk score alone (0.86 [0.75-0.97] versus 0.96 [0.92-1.0], $p=0.09$ ), and the highest AUC across all analyzed factors for six-month mortality was observed for the combination of copeptin with the GRACE risk score
(0.98 [0.95-1.0], $p<0.001)$. It outperformed both of the predictors alone $(p=0.04)$ and hs-TnT $(p<0.001)$.

In the Cox regression analysis, patients with copeptin level $\geq 17.4 \mathrm{pmol} / \mathrm{l}$ were at significantly higher risk of death at six months (hazard ratio (HR) $14(1.7-117), p=0.014)$ and at one year (HR 4.32 [1.27-14.77], $p=0.02)$. Contrary, copeptin level $\geq 10 \mathrm{pmol} / \mathrm{l}$ was not a significant risk factor for death at six months (HR 61 [0.1-26.86], $p=0.19$ ) nor at one year (HR 4.56 [0.99-21.11], $p=0.052$ ). Hs-TnT measured at admission was not a predictor of mortality in the Cox analysis, neither was maximal concentration of hsTnT. Statistical significance for the prediction of mortality was reached by age (HR 1.1 [1.04-1.18], $p=0.002)$ and GFR (HR 0.92 [0.89-0.95], $p<0.001$ ). Detailed results are presented in Table 2. The combination of higher copeptin/ older age/lower GFR identified a group with higher admission and follow-up NT-proBNP levels, lower baseline 
TABLE 2: The Cox regression model for death at 6 and 12 months.

\begin{tabular}{|c|c|c|c|c|c|c|}
\hline \multirow{2}{*}{ Characteristic } & \multicolumn{3}{|c|}{ At six months } & \multicolumn{3}{|c|}{ At one year } \\
\hline & HR & $95 \%$ CI & $p$ value & HR & $95 \% \mathrm{CI}$ & $p$ value \\
\hline Copeptin $\geq 17.4 \mathrm{pmol} / 1$ & 14.1 & $1.7-116.8$ & 0.01 & 4.3 & $1.3-14.8$ & 0.02 \\
\hline Age & 1.3 & $1.1-1.5$ & 0.001 & 1.1 & $1.04-1.2$ & 0.002 \\
\hline Male sex & 0.2 & $0.04-1.1$ & 0.06 & 0.6 & $0.2-1.9$ & 0.359 \\
\hline Diagnosis of NSTEMI & 3.0 & $0.4-24.7$ & 0.31 & 2.3 & $0.5-10.8$ & 0.281 \\
\hline Prior AMI & 1.0 & $0.2-5.0$ & 0.96 & 1.4 & $0.4-4.8$ & 0.591 \\
\hline Diabetes mellitus & 1.1 & $0.2-5.8$ & 0.89 & 1.1 & $0.3-4.1$ & 0.909 \\
\hline GFR & 0.9 & $0.86-0.95$ & $<0.001$ & 0.99 & $0.9-0.95$ & $<0.001$ \\
\hline $\mathrm{EF}$ & 1.0 & $0.9-1.0$ & 0.09 & 0.95 & $0.9-0.99$ & 0.026 \\
\hline Admission NYHA class 3 or 4 & 7.7 & $1.3-46.1$ & 0.03 & 11.8 & $1.9-72$ & 0.008 \\
\hline Hs-TnT $\geq 14 \mathrm{ng} / \mathrm{l}$ at admission & 33.6 & $0.03-366,658$ & 0.33 & 4.1 & $0.5-32.3$ & 0.176 \\
\hline Hs-TnT mx & 1.0 & $0.99-1.0$ & 0.82 & 1.0 & $0.99-1.0$ & 0.642 \\
\hline
\end{tabular}

AMI: acute myocardial infarction; CI: confidence interval; EF: ejection fraction; GFR: glomerular filtration ratio; GRACE: Global Registry of Acute Coronary Events; HR: hazard ratio; hs-TnT mx: maximal concentration of high-sensitive troponin T; NSTEMI: non-ST segment elevation myocardial infarction; NYHA: New York Heart Association.

ejection fraction, and higher risk according to the GRACE risk score (Supplemental Table S2).

\section{Discussion}

This prospective, observational study assessed the prognostic role of copeptin and its combination with hs-TnT in consecutive patients with acute chest pain admitted to the emergency department of a tertiary cardiologic center.

The first finding is the high prognostic accuracy of copeptin in the prediction of mortality. According to previously published data $[11,12]$, we report higher mortality in patients with higher plasma levels of copeptin. Secondly, copeptin had significantly better prognostic accuracy than hs-TnT in studied population. There was no benefit from a combined use of copeptin and hs-TnT over copeptin alone. Thirdly, copeptin should be considered together with other, but still simple risk factors, especially age and renal function, while assessing prognosis. Lastly, we provide indirect evidence for the significant prognostic value of copeptin in the prediction of HF at long-term assessed with NT-proBNP, a recently strongly recommended diagnostic parameter of HF [13].

The prognostic value of copeptin in patients with chest pain is the field of research interest and constant growth. In this study, we add evidence on prognostic utility of copeptin as an early marker of adverse outcome in a specific population of highly preselected patients with high rate of finally diagnosed ACS, characteristic for tertiary centers. The decision on optimal type and time frame of the management is essential for non-ST segment elevation ACS and appropriate selection of patients in whom invasive interventions are likely to be beneficial. According to the guidelines [1], the triage of patients in such circumstances should be extensive; involve clinical status of patients, medical history, the dynamics of ECG, and biomarker concentrations; and be supported by different risk scoring systems, for example, the GRACE risk score. Despite the GRACE risk score is a validated tool in risk stratification
[14], the practical utility is compromised in everyday clinical practice [15]. We provide evidence that supports very early risk assessment with single measure from a blood draw at admission. The results of the Cox analysis identified copeptin, age, and renal dysfunction as risk markers of poor prognosis at one year. Whether this combination of risk factors would play a wide practical role remains to be determined in a larger cohort.

The single measurement of copeptin at admission showed also a tendency to increase the prognostic accuracy of the GRACE risk score for death at six months. It confirms previous reports on good prognostic performance of copeptin in the prediction of death, however assessed in different clinical setting including STEMI [16] or derived from measurements at later time points [6]. Nevertheless, these results might serve as a background for further research on a simple and concise prognostic evaluation methodology, which would be of clinical benefit at the bedside, without the need for extensive, repetitive examinations or online calculations.

The combination of copeptin and hs-TnT merits consideration. The estimation of prognosis based on combined use of copeptin and hs-TnT was not clinically relevant. Surprisingly, hs-TnT alone was also not a significant predictor of long-term death. It is known that cTn directly correlates with infarct size [17] and the predictive value of cTn is higher for maximal concentrations measured during the hospital stay [18]. Of note is a correlation found for copeptin with maximal concentrations of hs-TnT in our study. This leads to speculate, first, that this is the reason why hs-TnT measured at admission had low prognostic value and, second, that copeptin might be considered an early indirect predictor of infarct size. The latter hypothesis was recently evaluated and confirmed in a population of STEMI patients [19]. As a consequence might be regarded the observation of predictive value of copeptin for the development of heart failure, with higher and diagnostic for HF levels of NT-proBNP among patients stratified with copeptin, age, and GFR as high risk. It was not reflected in the value of EF but it is known that levels of NT-proBNP might be elevated also in 
patients with preserved EF [20]. We would like, however, to prevent the reader from interpreting our results as a prove of direct relationship between copeptin and infarct size and/or heart failure and consider them as a hypothesis that warrants further research.

In conclusion, copeptin appears to be an independent predictor of long-term mortality in a selected population of patients suspected for an ACS. In addition, copeptin may be considered as an early marker for the identification of patients at higher risk of the development of HF at long term in this population. The outcomes warrant a confirmation in a larger cohort.

4.1. Limitations. The following limitations should be mentioned. The observational character of the study limits the interpretation of clinical benefit from risk assessment with copeptin. Further, studied troponin was the hs-TnT (Roche). It remains unknown if the use of other assays or other troponin would influence the outcomes. Next, in the study, we used a prespecified cutoff for copeptin at the 97.5th percentile, as the closest to that recommended by the guidelines (99th percentile) that was available from the manufacturer's resources; therefore, the outcomes are limited with regard to previously proposed values $[6,11]$. Further studies are warranted to directly compare different cutoffs to identify the optimum for prognostic purposes.

\section{Disclosure}

Drs. Beata Morawiec and Damian Kawecki are considered the first authors.

\section{Conflicts of Interest}

The authors have no conflict of interest to disclose.

\section{Authors' Contributions}

Beata Morawiec and Damian Kawecki contributed equally to this publication.

\section{Supplementary Materials}

Table S1: baseline characteristics in patients positive and negative for copeptin with the cutoff $10 \mathrm{pmol} / \mathrm{l}$. Table S2: outcomes of the taxonomic analysis. Figure S1: survival curves or copeptin with the cutoff $10 \mathrm{pmol} / \mathrm{l}$. Figure S2: dendrogram - taxonomic analysis. (Supplementary Materials)

\section{References}

[1] M. Roffi, C. Patrono, J. P. Collet et al., "2015 ESC guidelines for the management of acute coronary syndromes in patients presenting without persistent ST-segment elevation: task force for the management of acute coronary syndromes in patients presenting without persistent ST-segment elevation of the European Society of Cardiology (ESC)," European Heart Journal, vol. 37, no. 3, pp. 267-315, 2016.

[2] K. Thygesen, J. S. Alpert, H. D. White, and Joint ESCAAHAWHFTFftRoMI, "Universal definition of myocardial infarction," European Heart Journal, vol. 28, no. 20, pp. 2525-2538, 2007.

[3] M. L. O'Donoghue, D. A. Morrow, C. P. Cannon et al., "Multimarker risk stratification in patients with acute myocardial infarction," Journal of the American Heart Association, vol. 5, no. 5, article e002586, 2016.

[4] B. Morawiec and D. Kawecki, "Copeptin: a new marker in cardiology," Journal of Cardiovascular Medicine, vol. 14, no. 1, pp. 19-25, 2013.

[5] C. T. Lui, H. Lam, K. H. Cheung et al., "Role of copeptin in dual-cardiac marker strategy for patients with chest pain presented to ED," The American Journal of Emergency Medicine, vol. 33, no. 12, pp. 1732-1736, 2015.

[6] S. Q. Khan, O. S. Dhillon, R. J. O'Brien et al., "C-terminal provasopressin (copeptin) as a novel and prognostic marker in acute myocardial infarction: Leicester Acute Myocardial Infarction Peptide (LAMP) study," Circulation, vol. 115, no. 16, pp. 2103-2110, 2007.

[7] T. Reichlin, W. Hochholzer, C. Stelzig et al., "Incremental value of copeptin for rapid rule out of acute myocardial infarction," Journal of the American College of Cardiology, vol. 54, no. 1, pp. 60-68, 2009.

[8] A. A. Voors, S. von Haehling, S. D. Anker et al., "C-terminal provasopressin (copeptin) is a strong prognostic marker in patients with heart failure after an acute myocardial infarction: results from the OPTIMAAL study," European Heart Journal, vol. 30, no. 10, pp. 1187-1194, 2009.

[9] B. Morawiec, D. Kawecki, L. Ho, L. Chun Tat, O. Muller, and E. Nowalany-Kozielska, "COPeptin for diagnosis and prediction in Acute Coronary Syndrome (COPACS) Study: design and objectives," Advances in Interventional Cardiology, vol. 12, no. 46, pp. 1-4, 2016.

[10] M. Mockel, J. Searle, C. Hamm et al., "Early discharge using single cardiac troponin and copeptin testing in patients with suspected acute coronary syndrome (ACS): a randomized, controlled clinical process study," European Heart Journal, vol. 36, no. 6, pp. 369-376, 2015.

[11] C. Zellweger, K. Wildi, R. Twerenbold et al., "Use of copeptin and high-sensitive cardiac troponin $\mathrm{T}$ for diagnosis and prognosis in patients with diabetes mellitus and suspected acute myocardial infarction," International Journal of Cardiology, vol. 190, pp. 190-197, 2015.

[12] C. Balmelli, C. Meune, R. Twerenbold et al., "Comparison of the performances of cardiac troponins, including sensitive assays, and copeptin in the diagnostic of acute myocardial infarction and long-term prognosis between women and men," American Heart Journal, vol. 166, no. 1, pp. 30-37, 2013.

[13] P. Ponikowski, A. A. Voors, S. D. Anker et al., "2016 ESC guidelines for the diagnosis and treatment of acute and chronic heart failure: the task force for the diagnosis and treatment of acute and chronic heart failure of the European Society of Cardiology (ESC) developed with the special contribution of the Heart Failure Association (HFA) of the ESC," European Heart Journal, vol. 37, no. 27, pp. 21292200, 2016.

[14] F. D'Ascenzo, G. Biondi-Zoccai, C. Moretti et al., "TIMI, GRACE and alternative risk scores in acute coronary syndromes: a meta-analysis of 40 derivation studies on 216,552 patients and of 42 validation studies on 31,625 patients," Contemporary Clinical Trials, vol. 33, no. 3, pp. 507-514, 2012. 
[15] O. Manfrini and R. Bugiardini, "Barriers to clinical risk scores adoption," European Heart Journal, vol. 28, no. 9, pp. 10451046, 2007.

[16] R. G. O'Malley, M. P. Bonaca, B. M. Scirica et al., "Prognostic performance of multiple biomarkers in patients with nonST-segment elevation acute coronary syndrome," Journal of the American College of Cardiology, vol. 63, no. 16, pp. 16441653, 2014.

[17] V. C. Vasile, L. Babuin, E. Giannitsis, H. A. Katus, and A. S. Jaffe, "Relationship of MRI-determined infarct size and cTnI measurements in patients with ST-elevation myocardial infarction," Clinical Chemistry, vol. 54, no. 3, pp. 617-619, 2008.

[18] T. Reynolds, M. Cecconi, P. Collinson, A. Rhodes, R. M. Grounds, and M. A. Hamilton, "Raised serum cardiac troponin I concentrations predict hospital mortality in intensive care unit patients," British Journal of Anaesthesia, vol. 109, no. 2, pp. 219-224, 2012.

[19] V. Ananth, J. R. Beig, N. A. Tramboo et al., "Does plasma copeptin level at admission predict final infarct size in STelevation myocardial infarction," International Journal of Cardiology, vol. 219, pp. 326-330, 2016.

[20] A. Maisel, C. Mueller, K. Adams et al., "State of the art: using natriuretic peptide levels in clinical practice," European Journal of Heart Failure, vol. 10, no. 9, pp. 824-839, 2008. 


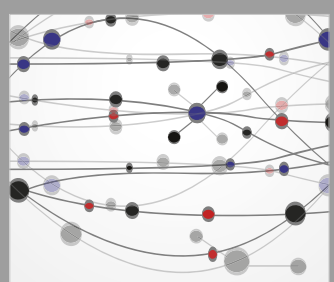

The Scientific World Journal
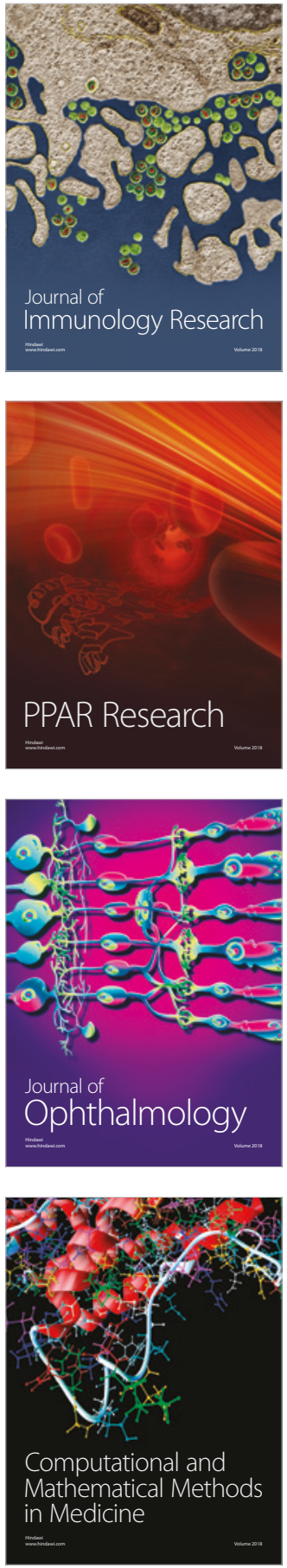

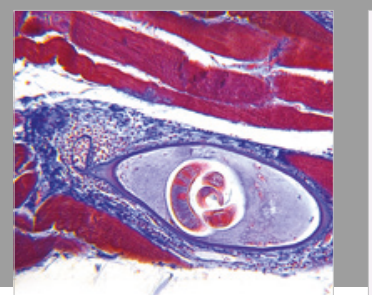

Gastroenterology Research and Practice

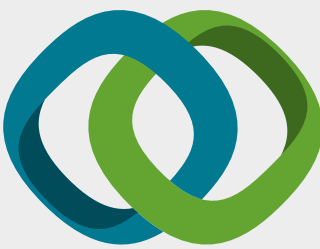

\section{Hindawi}

Submit your manuscripts at

www.hindawi.com
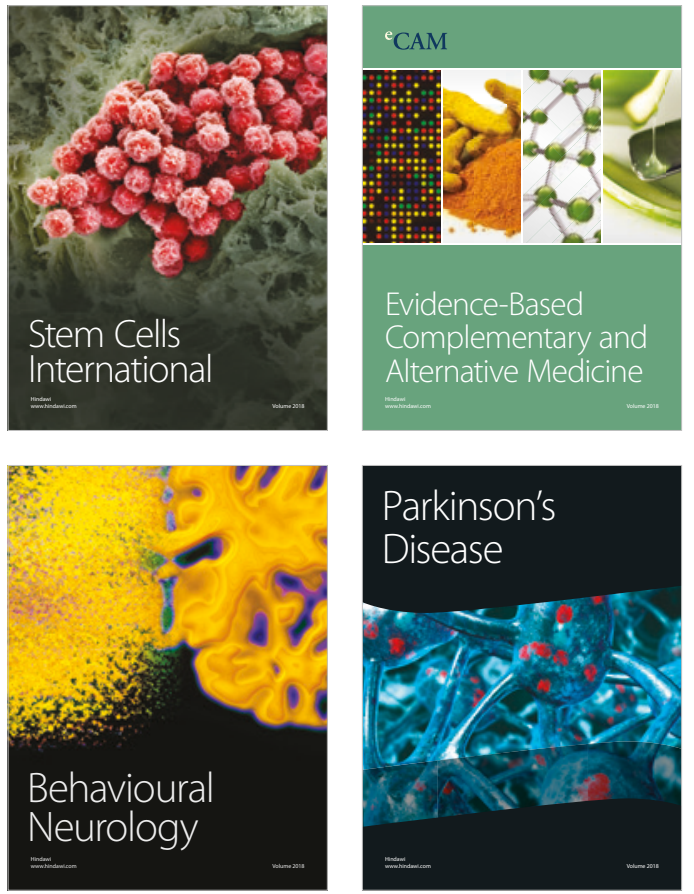

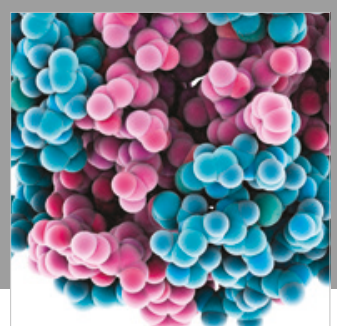

ournal of

Diabetes Research

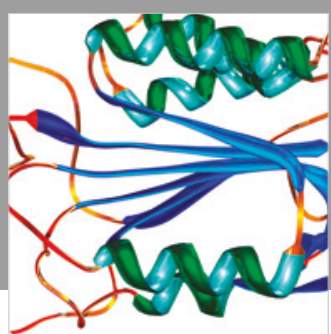

Disease Markers
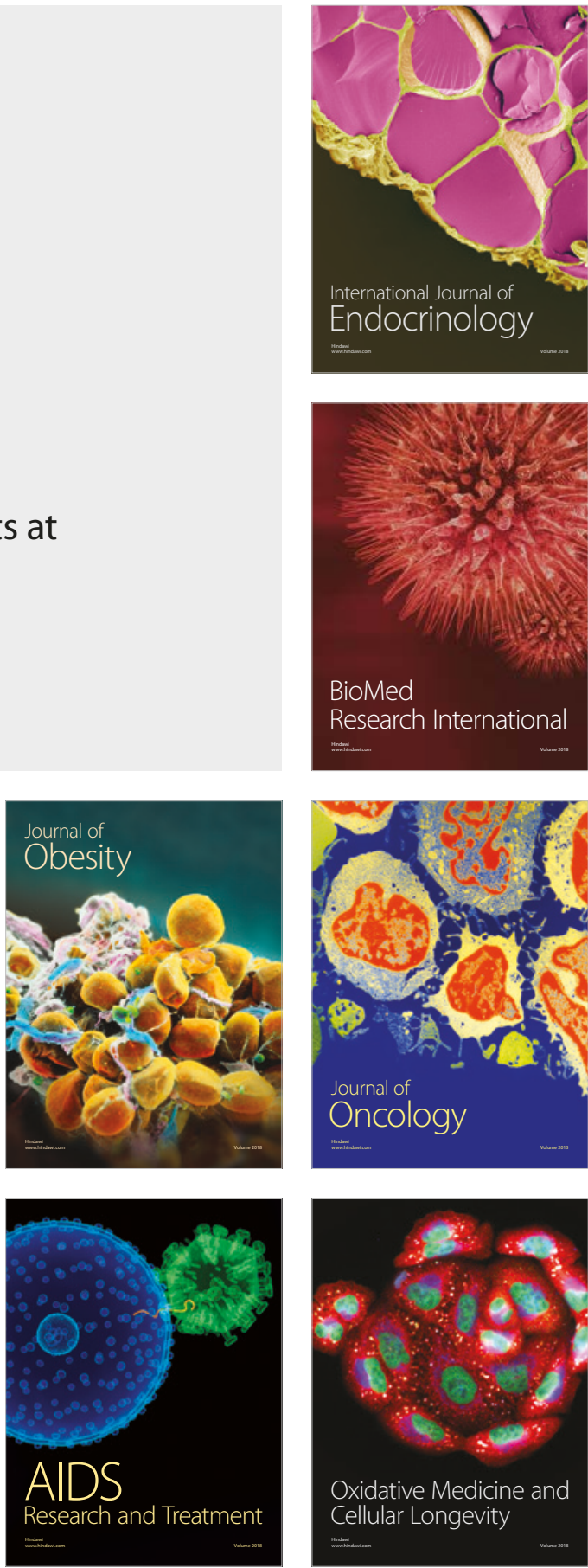